\title{
First-principles Calculations of Complex Intermediate Band Materials for Photovoltaic Devices
}

\author{
P. Wahnón ${ }^{1}$, I. Aguilera ${ }^{1}$, P. Palacios ${ }^{1}$ and K. Sánchez ${ }^{1}$ \\ ${ }^{1}$ Instituto de Energía Solar \& Dept. Tecnologías Especiales, Universidad Politécnica de Madrid, \\ Ciudad Universitaria s/n, Madrid, 28040, Spain.
}

\begin{abstract}
An $a b$ initio study of several compounds candidates to behave as intermediate band materials is presented. The use of these materials as the active element in solar cells is a promising way to enhance the photovoltaic efficiency. Indeed from this point of view, most interesting compounds are those whose host semiconductor presents a band-gap close to the optimum value of $2 \mathrm{eV}$. Chalcogenide compounds substituted by light transition metals are solid candidates to this end. While they are being further characterized and experimentally synthesized, another approach is being examined. It consists of using $\mathrm{Si}$ as host semiconductor. Ti implantation at concentrations several orders of magnitude above equilibrium solubility has shown a probable intermediate band material behavior, the origin of the intermediate band being related to levels of interstitial Ti. Optoelectronic characterization of this material is completed. A novel possibility consists of combining chalcogen $S$ implantation with boron. In this case preliminary results of electronic structure are shown.
\end{abstract}

\section{INTRODUCTION}

Intermediate band solar cell concept [1] is enclosed in the so-called third generation of solar cells. It is based on the electronic structure of the absorber material, which presents a narrow, partially-filled intermediate band. This band helps to enhance the photocurrent with regards to a host semiconductor, but leaving unchanged the photovoltage. The additional photocarriers are generated in a double-step process involving the intermediate band due to photons with energies below band-gap can be used to pump electrons from valence band to the intermediate band or from the latter to the conduction band.

A number of materials with substitution of atoms by transition metals have been proposed in past years [2-5]. Chalcogenide-derived compounds are particularly interesting today due to their use as thin-film materials in the photovoltaic area. Furthermore, use of thin-film techniques would allow for these materials to reduce the costs of the solar cell. Experimental measurements of defective thiospinel $\operatorname{In}_{2} \mathrm{~S}_{3}$ with replacement of In by transition metals [6] have shown the characteristic triple absorption spectra predicted by quantum calculations [7]. Growth of larger samples for this compound and other chalcogenide-derived materials are underway.

However materials based on $\mathrm{Si}$ have been proposed in order to achieve in a short-term an intermediate band solar cell with enhanced efficiency. Si implanted with Ti at concentrations close to $10^{20} \mathrm{~cm}^{-3}$ was the first candidate [8]. The intermediate band was explained to be formed from the $t_{2 g}$ type manifold of $3 \mathrm{~d}$ electrons of Ti atoms placed in tetrahedral interstitials [9]. While 
optical characterization of the experimental samples has not been yet carried out, electrical measurements points towards an intermediate band formation [10].

In this communication further results of optical properties are carried out, in order to support the comparison with future experimental results. Besides, preliminary results of a possible candidate formed by combination of sulfur implantation and group IIIB elements, as B, are presented.

\section{THEORY}

Quantum calculations carried out in this study follow Density Functional Theory (DFT) $[11,12]$ implemented in the plane-wave based VASP package[13,14]. Exchange-correlation potential was evaluated by means of the Generalized Gradient Approximation (GGA), parameterized through PW91 functional [15]. Projector Augmented Wave (PAW) potentials [16] were used in order to interpret the core electrons. Brillouin zone was sampled by a $4 \times 4 \times 4$ Monkhorst-Pack grid of k-points including $\Gamma$ point in the calculations.

Cells used in all the calculations are derived from a $3 \times 3 \times 3$ supercell of conventional $\mathrm{Si}_{8}$ cell. In the case of Ti implanted $\mathrm{Si}$, relaxation of ions and lattice was carried out until obtaining a tolerance of $0.01 \mathrm{eV} / \AA \hat{\text {. }}$.

Optical properties were determined from the dielectric function which, in turn, was calculated through the Fermi's golden rule, by carrying out a sum over independent transitions between Kohn-Sham states. Only direct transitions were taken into account. Calculations were carried out using the OPTICS code $[17,18]$. In order to achieve convergence over optical properties an 8x8x8 grid of reciprocal lattice was used and 800 bands were included.

\section{DISCUSSION}

\section{$\underline{\text { Ti-implanted Si optical properties }}$}

Donor-level found for Ti implanted Si has been theoretically related to a transition involving an intermediate band formed by $t_{2 g}$ electrons of interstitial Ti [9]. However, no direct measurement of the intermediate band has been reported so far.

In a previous work, absorption coefficient of $\mathrm{Ti}_{\mathrm{i}} \mathrm{Si}_{216}$ compound (therefore showing a $\mathrm{Ti}$ concentration in the order of magnitude of $10^{20} \mathrm{~cm}^{-3}$ ) was calculated from dielectric function and compared with that of bulk $\mathrm{Si}_{216}$ showing an enhancement across the solar spectrum [9]. Nevertheless, from the experimental point of view other magnitudes are preferred. A property that is usually obtained experimentally is reflectance $(R)$ which is related with the refractive index $(n)$ and the extinction coefficient $(\kappa)$ through the formula:

$$
R=\frac{(n-1)^{2}+\kappa^{2}}{(n+1)^{2}+\kappa^{2}}
$$


However, as it can be seen in figure 1, the difference between reflectance of bulk $\mathrm{Si}_{216}$ and $\mathrm{Ti}_{\mathrm{i}} \mathrm{Si}_{216}$ is not significant. For this reason identifying an intermediate band focusing only on reflectance measurements seems a difficult task.

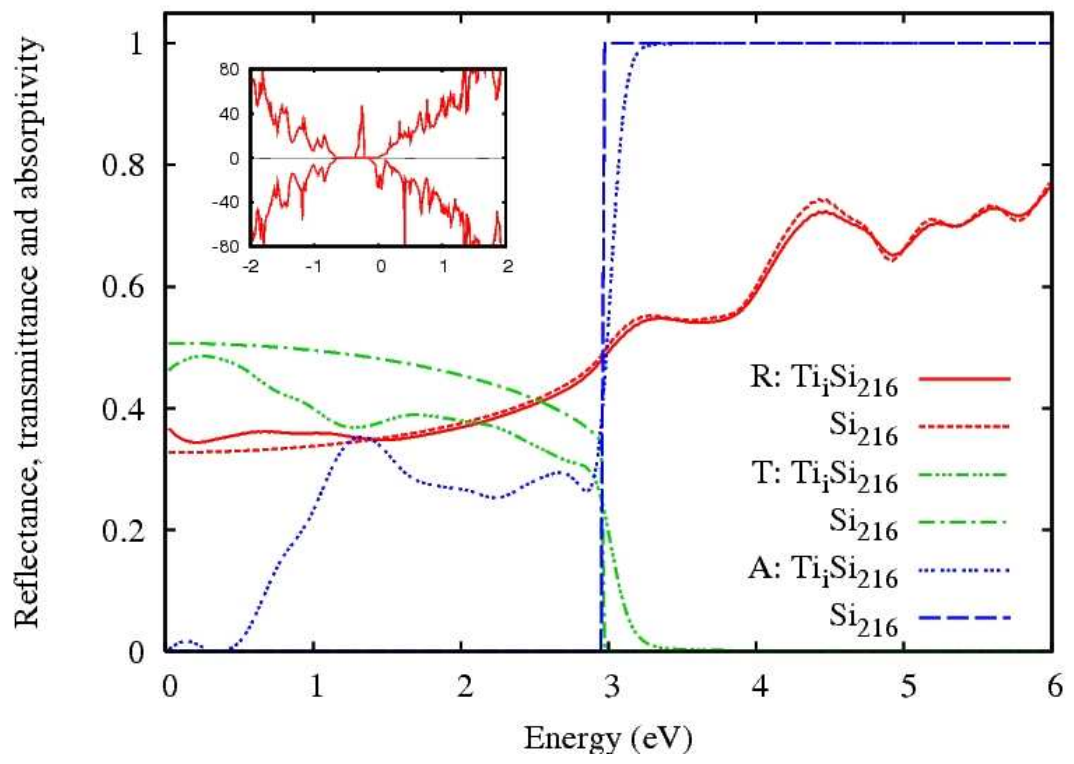

Figure 1. Reflectance, transmittance, and absorptivity calculated for $\mathrm{Ti}_{\mathrm{i}} \mathrm{Si}_{216}$ (considering a sample thickness of $50 \mathrm{~nm})$ and bulk $\operatorname{Si}_{216}(300 \mu \mathrm{m}$ thickness). Density of states of the intermediate band material is shown in the inset.

Conversely, transmittance is a feature more sensible to the Ti insertion. It can be written as:

$$
T=\frac{(1-R)^{2} e^{-\alpha d}}{1-R^{2} e^{-2 \alpha d}}
$$

Where $\alpha$ is the absorption coefficient, and $d$ is the thickness of the absorber. In figure $1 \mathrm{a}$ thickness of $50 \mathrm{~nm}$ of Ti-implanted material is used to reproduce the value assumed in the sample [10]. As a reference, a bulk Si thickness of $300 \mu \mathrm{m}$, usual in wafers, is also considered. It is worth mentioning that variation of the width of Si sample produces only minor changes in the properties represented in figure 1. Contrast between both transmittances is observed especially at low energies.

Another clear differentiation between intermediate band material and semiconductor results corresponds to absorptivity of the sample, which is defined here as:

$$
A=1-e^{-2 \alpha d}
$$

Indeed, calculated absorptivity of $\mathrm{Ti}_{\mathrm{i}} \mathrm{Si}_{216}$ for energies between 1 and $3 \mathrm{eV}$ shows a value of 0.3 approximately. It is well known that indirect transitions, at least for bulk Si, would produce a tail in the absorption at that energy range. Despite they are absent in the present calculations there is still another energy range below the indirect band-gap in which the intermediate band material would show a non-null absorptivity, conversely to the case of bulk Si. 


\section{$\underline{\text { S-implanted Si and co-doped with B }}$}

Electronic density of states of S-implanted $\mathrm{Si}$ with substitution of one $\mathrm{Si}$ atom by one $\mathrm{S}$ is represented in figure 2. Substitution is considered the most common configuration of $\mathrm{S}$ impurities in $\mathrm{Si}$ [19] and corresponds to $\mathrm{SSi}_{215}$ unit cell. This concentration level of $0.5 \%$ has been already achieved in the laboratory [20]. In figure 2 a scissor operator of $0.5 \mathrm{eV}$ has been applied to the empty states in order to correct the well-known bandgap underestimation carried out in GGA calculations. As a result, a narrow band appears within the semiconductor energy band-gap. This band is originated from the replacement of a Si atom by sulfur and is completely occupied, which is linked with the double-donor character of this kind of impurity. For that reason a co-doping is suggested in order to reduce the electronic occupation of the band.

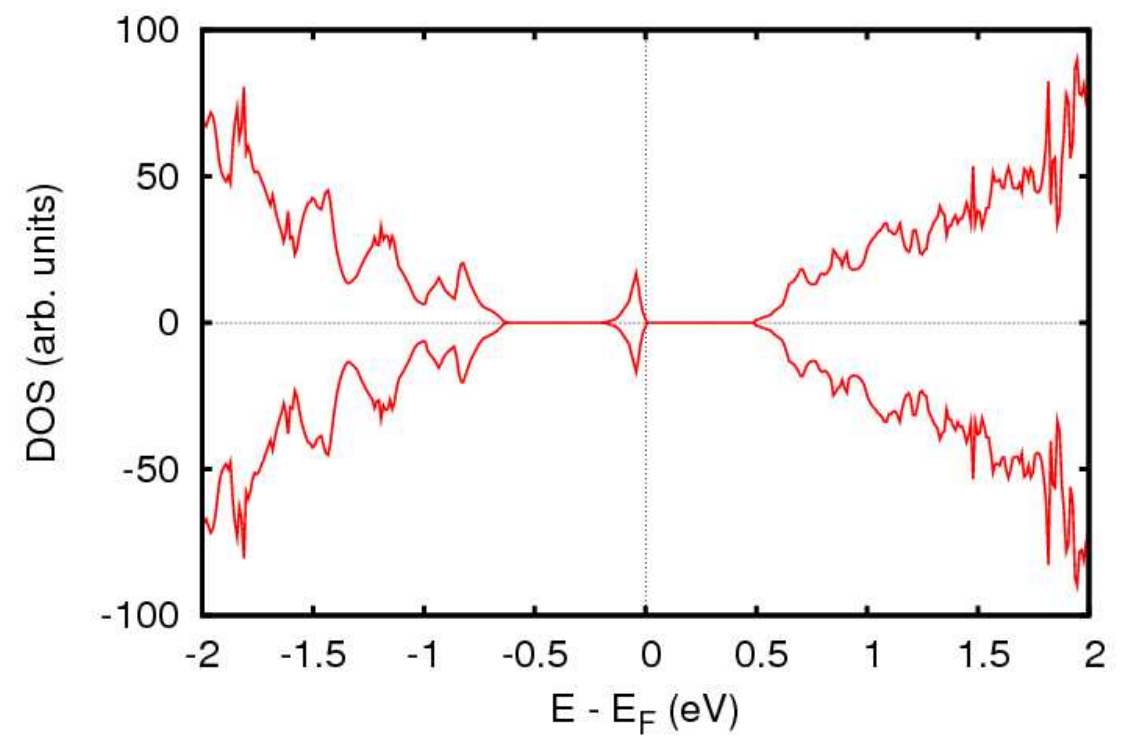

Figure 2. Density of states of $\mathrm{S}_{\mathrm{Si}} \mathrm{Si}_{215}$ compound. Upper (lower) part corresponds to majority (minority) spin states. Fermi level is located at zero energy in the figure.

Calculation of the electronic structure for a co-doping of S with $\mathrm{B}$ over $\mathrm{Si}$ at a concentration of approximately $0.5 \%$ (that is, $2 \times 10^{20} \mathrm{~cm}^{-3}$ ) has been carried out, using again the experimental lattice parameter of Si. Boron can also be introduced in $\mathrm{Si}$ at this degree of concentration in $\mathrm{Si}$ [21]. A homogeneous arrangement of dopants has been considered in the calculation. The resulting density of states is shown in figure 3. It can be seen a half-filled intermediate band located within the host-semiconductor band-gap. Although a correction for the underestimation of the semiconductor band-gap has not been considered in figure 3, that correction would not introduce a qualitative discrepancy but only would further increase the energetic difference between the bands. 


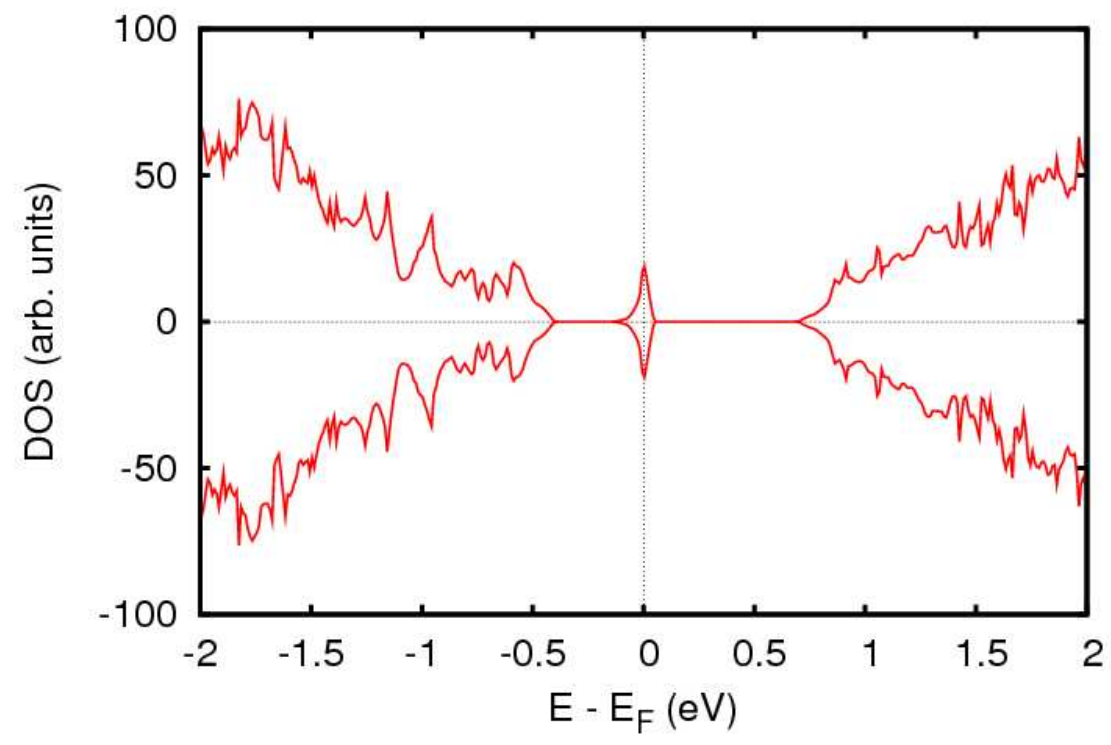

Figure 3. Density of states of $\mathrm{B}_{\mathrm{Si}} \mathrm{S}_{\mathrm{Si}} \mathrm{Si}_{214}$ compound.

Substitutional S is behaves as a deep double-donor in Si and when combined with the shallow-acceptor character of substitutional B, the additional hole of the latter is transferred to the band lying in the gap, therefore producing the intermediate band shown in figure 3 . Although a deeper study is needed to improve the knowledge about $\mathrm{B}_{\mathrm{Si}} \mathrm{S}_{\mathrm{Si}_{\mathrm{i}}} \mathrm{Si}_{214}$, this compound is a potential intermediate band material.

\section{CONCLUSIONS}

Optical properties susceptible of being measured experimentally for Si implanted with $\mathrm{Ti}$ occupying interstitials have been calculated and compared with those of bulk-Si. Whereas reflectance seems not to be a feature particularly useful to make a distinction between the intermediate band material and its parent semiconductor, both transmittance and absorptivity are expected to enhance the differences between these compounds.

Additionally, preliminary calculations show an intermediate band in the electronic structure calculated for $\mathrm{B}_{\mathrm{Si}} \mathrm{S}_{\mathrm{Si}} \mathrm{Si}_{214}$. In order to get a deeper insight, an intensive study of this compound and other combinations of chalcogens and group IIIB elements substituting Si atoms is underway.

\section{ACKNOWLEDGMENTS}

Authors would like to thank funding from projects GENESIS-FV (CSD2006-2004) and FOTOMAT (MAT2009-14625-CO3-01). Computer resources and assistance provided by Centro de Supercomputación y Visualicación de Madrid (CeSViMa) are also acknowledged. I.A. would also to thank the MEC for a FPU grant and K.S. acknowledges a grant from Com. de Madrid and European Social Fund. 


\section{REFERENCES}

1. A. Luque, A. Martí, Phys. Rev. Lett. 78, 5014 (1997).

2. P. Wahnón, C. Tablero, Phys. Rev. B 65, 165115 (2002).

3. P. Palacios, J. J. Fernández, K. Sánchez, J. C. Conesa, and P. Wahnón, Phys. Rev. B 73, 085206 (2006).

4. P. Palacios, K. Sánchez, J. C. Conesa, P. Wahnón, Phys. Stat. Solidi (a) 203, 1395 (2006).

5. P. Palacios, K. Sánchez, J. C. Conesa, J. J. Fernández, P. Wahnón, Thin Solid Films 515, 6280 (2007).

6. R. Lucena, I. Aguilera, P. Palacios, P. Wahnón, J. C. Conesa, Chem. Maters. 20, 512 (2008).

7. P. Palacios, I. Aguilera, K. Sánchez, J. C. Conesa, P. Wahnón, Phys. Rev. Lett. 101, 046403 (2008).

8. J. Olea, M. Toledano-Luque, D. Pastor, G. González-Díaz, I. Mártil, J. Appl. Phys. 104, 016105 (2008).

9. K. Sánchez, I. Aguilera, P. Palacios, P. Wahnón, Phys. Rev. B 79, 165203 (2009).

10. J. Olea, G. González-Díaz, D. Pastor, I. Mártil, J. of Phys D: Appl. Phys. 42, 085110 (2009).

11. P. Hohenberg, W. Kohn, Phys. Rev. 136, B864 (1964).

12. W. Kohn, L. J. Sham, Phys. Rev. 140, A1133 (1965).

13. G. Kresse, J. Hafner, Phys. Rev. B 47, 558 (1993).

14. G. Kresse, J. Furthmüller, Phys. Rev. B 54, 11169 (1996).

15. J. P. Perdew, J. A. Chevary, S. H. Vosko, K. A. Jackson, M. R. Pederson, D. J. Singh, C. Fiolhais, Phys. Rev. B 46, 6671 (1992).

16. G. Kresse, D. Joubert, Phys. Rev. B 59, 1758 (1999).

17. M. Gajdos, K. Hummer, G. Kresse, J. Furthmüller, and F. Bechstedt, Phys. Rev. B 73, $045112(2006)$.

18. J. Furthmüller, computer code OPTICS, http://www.freeware.vasp.de/VASP/optics/ (At the date this paper was written, URL referenced code and information. Neither the authors nor the MRS warrants or assumes liability for the content or availability of URL referenced).

19. H. Overhof, M. Scheffler, C.M. Weinert, Phys. Rev. B 91, 12494 (1991).

20. M. Tabbal, T. Kim, J. M. Warrender, M.J. Aziz, B.L. Cardozo, R.S. Goldman, J. Vac. Sci. Technol. B 25, 1847 (2007).

21. R.W. Olesinski, and G.J. Abbaschian, Bull. Alloy Phase Diagrams 5, 478 (1984). 